\section{P238 CHARACTERISTICS, TREATMENT PATTERNS AND OUTCOMES OF PATIENTS WITH NON-CF BRONCHIECTASIS: A SINGLE INSTITUTION DISTRICT GENERAL HOSPITAL (DGH) ANALYSIS}

doi:10.1136/thx.2010.151068.39

M J McDonnell, C Mashamba, A Foden. County Durham and Darlington NHS Foundation Trust, Darlington, Tyne and Wear, UK

Introduction Non-CF bronchiectasis has been the subject of analysis for several years with limited guidelines available regarding appropriate investigation and management strategies to optimise patient care. Non-CF bronchiectasis is common and, unlike CF, is often managed by general respiratory physicians in a DGH setting. BTS consensus, based largely on case-control series and cohort studies, has recently been published to aid clinicians in diagnosis and management. Objectives The aim of this retrospective study is to present data on patient characteristics, treatment patterns, and treatment results in an unselected patient group with non-CF bronchiectasis over a 9year period.

Methods From January 2000 to December 2009, we reviewed the clinical, radiological, microbiological, and physiological findings in 73 well-studied patients with proven non-CF bronchiectasis. We collected data on drug and non-drug management, including side effects and response to treatment-measured as improvement in pulmonary lung function (PFTs).

Results There was a male:female ratio of $1: 2$ with mean age of 51.4 years (range 3-81); 46.6\% were lifetime non-smokers. Idiopathic bronchiectasis was confirmed in $54.7 \%$ patients on completion of full bronchial sepsis screen. Of the idiopathic group, $42.5 \%$ were smokers; $22.5 \%$ of these were confirmed to have COPD prior to diagnosis of bronchiectasis. HRCT confirmed diagnosis of bronchiectasis in $82.2 \%$ of patients with bibasal predominance in majority. Initial CXR was abnormal in $62.8 \%$. PFTs documented airway obstruction in $54 \%$ of lifetime non-smokers. Smokers had greater degree of airway obstruction than non-smokers and greater number of exacerbations/ patient/year. Pathologic microbial flora isolated from sputum included Haemophilus influenza and other opportunistic organisms. $17.8 \%$ patients were colonised with Pseudomonas aeruginosa and treated with prophylactic nebulised antibiotics. There was no relationship between COPD and pseudomonas colonisation. $5.5 \%$ patients were treated with prophylactic oral antibiotics. Side-effects occurred in $4.1 \%$ overall (Clostridium difficile). Factors contributing to worsening of PFTs include increased number of exacerbations/patient/year, pseudomonas colonisation and smoking status.

Conclusion We provide a comprehensive analysis of a contemporary patient population. Treatment patterns fit well in the context of current consensus based on international trials. We suggest a likely correlation between the pathophysiology of COPD and bronchiectasis which warrants further investigation with randomised controlled trials.

\section{P239 AUDIT OF ONCE DAILY NEBULISED HYPERTONIC 6\% SALINE (HTS) IN ADULT NON-CF BRONCHIECTASIS}

doi:10.1136/thx.2010.151068.40

H Pyne, B Kane, B R O'Driscoll. Salford Royal Foundation hospital Trust, Salford, UK

Background Nebulised hypertonic $7 \%$ saline enhances sputum clearance in patients with bronchiectasis and hypersecretion (Kellet F et al Med 2005; 99:27-31) but is not licensed for this purpose. It is expensive to produce, has a short shelf life and is difficult to administer. Mucoclear ${ }^{\circledR} 6 \%$ saline does not have these disadvantages so we wished to evaluate its clinical benefits.
Method Patients with troublesome bronchiectasis were invited for a nebulised $6 \%$ HTS challenge. If no adverse reaction occurred, they administered HTS daily for 2 months

Results 60 patients were assessed over 18 months. Ten had bronchospasm after HTS, 9 did not wish to continue treatment and 41 reported an initial positive response and administered HTS once daily for 2 months. All 41 patients reported improved ease of sputum clearance with a median 3 point increase on a 10 unit Viausal Analogue Scale. 49\% reported increase in sputum volume. 10 patients had baseline oxygen saturation $\leq 95 \%$ with $\geq 2 \%$ rise on HTS. $59 \%$ reported an increase in quality of life on Juniper mini asthma quality of life questionnaire (mean 0.6 unit rise overall and 1.0 for responders; rise of $>0.5$ units is significant). Mean $\mathrm{FEV}_{1}$ rose $9.5 \%$ (from 1.68 to 1.84 litres) and mean FVC rose $10.5 \%$ (from 2.48 to 2.74 litres). (Wilcoxon test $p<0.001$ ). There was no change in spirometry for seven patients with normal baseline measurements but $\mathrm{FEV}_{1}$ rose by $13 \%$ and FVC rose by $12 \%$ among 34 patients with abnormal lung function. All patients reported a noticeable improvement in their condition (12\% reported "life changing improvement").

Conclusion Despite standard therapies some adults with bronchiectasis have persistent troublesome hypersecretion. Two thirds of our patients reported a significant improvement in symptoms using HTS and, for some, this was life changing. This suggests that Mucoclear ${ }^{\circledR} 6 \%$ hypertonic nebulised saline is a viable option but controlled trials are needed.

\section{P240 LONGITUDINAL STUDY OF SPUTUM MICROBIOLOGY IN ADULT NON-CF BRONCHIECTASIS}

doi:10.1136/thx.2010.151068.4

${ }^{1} \mathrm{~J}$ G Macfarlane, ${ }^{2} \mathrm{P}$ McAlinden, ${ }^{3} \mathrm{~A}$ De Soyza. ${ }^{1}$ Freeman Hospital Respiratory Department, Newcastle Upon Tyne, UK; ${ }^{2}$ William Leech Clinical Research Centre Newcastle upon Tyne, UK; ${ }^{3}$ Institute of Cellular Medicine, Newcastle University, Newcastle upon Tyne, UK

Introduction and Objectives Monitoring longitudinal sputum microbiology in adults with non-CF bronchiectasis (nCF-Br) is a key strategy in guiding targeted antibiotic therapy. There is minimal published data on the microbiological profile over time in bronchiectasis. ${ }^{12}$ Similar to CF, greater pathogen diversity is now being observed; hence we have revisited this area

Methods 12 years of previous sputum culture results obtained from $143 \mathrm{nCF}-\mathrm{Br}$ patients attending a specialist clinic were retrospectively reviewed. 'Colonisation' (organism cultured $\geq 2$ occasions, 3 months apart within 1-year period) and 'isolation' (organism cultured $\geq 1$ ) were recorded.

Results $88 \mathrm{~F}$, $55 \mathrm{M}$ patients; average age 60.6 (range 16-90); average $\mathrm{FEV}_{1} 65 \%$ predicted (SD $\pm 26 \%$ ). The most common pathogens were Haemophilus influenzae (52\% isolated and 33\% colonisation; $8 \%$ were beta-lactam producing) and Pseudomonas aeruginosa (43\% isolated and $35 \%$ colonisation) whilst $20 \%$ patients had no pathogens cultured. 81 patients (57\%) have never had Pseudomonas. Of 62 patients (43\%) isolating Pseudomonas, 12 patients (8\%) had single isolates, $8(6 \%)$ had colonisation with successful eradication therapy. Streptococcus pneumoniae (34\%), Coliforms (30\%), Moraxella catarrhalis (27\%), Staphylococcus aureus (24\%) were other common isolates. Rarer pathogens include Aspergillus sp. (9\%), S.maltophilia (8\%), non-tuberculous mycobacteria (NTM 3\%; M. terrae, $M$. avium and $M$. simiae), MRSA(3\%), Acinetobacter sp. (3\%) and Achromobacter xylosoxidans (3\%).

Conclusions We note similar rates of $H$. influenzae colonisation as previously $\left(33 \%\right.$ vs $40 \%{ }^{1}$ ) but higher rates of Pseudomonas colonisation $\left(35 \%\right.$ vs $18 \%{ }^{1}$ and $\left.24 \%^{2}\right)$. Including those with any prior Pseudomonas, the rates of Pseudomonas isolation reach as high as $43 \%$ (higher than reported at $31 \%{ }^{2}$ ). Our unit receives referrals from the local immunodeficiency centre and other respiratory 